\title{
MRI-detected osteophytes of the knee: natural history and structural correlates of change
}

\author{
Zhaohua Zhu' ${ }^{1,2}$, Changhai Ding ${ }^{1,2,3}$, Weiyu Han ${ }^{1}$, Shuang Zheng ${ }^{2}$, Tania Winzenberg ${ }^{2,4}$, Flavia Cicuttini ${ }^{3}$
} and Graeme Jones ${ }^{2^{*}}$

\begin{abstract}
Backgroud: The natural history of semi-quantitative magnetic resonance imaging (MRI)-detected osteophytes (MRI-detected OPs) has not been described and it is unknown whether knee structural abnormalities can predict MRI-detected OP change over time. Thus, the aim of current study is to describe the natural history of knee MRI-detected OP, and to determine if knee structural abnormalities are associated with change of MRI-detected OP in a longitudinal study of older adults.
\end{abstract}

Methods: Randomly selected older adults ( $n=837$, mean age 63 years) had MRI at baseline and 413 of them had MRI 2.6 years later to measure MRI-detected OP, cartilage defects, cartilage volume, bone marrow lesions (BMLs), meniscal extrusion, infrapatellar fat pad (IPFP) quality score/maximum area and effusion-synovitis.

Results: Over 2.6 years, average MRI-detected OP score increased significantly in all compartments. The total MRIdetected OP score remained stable in 53\% of participants, worsened ( $\geq 1$-point increase) in $46 \%$ and decreased in $1 \%$. Baseline cartilage defects (RR, 1.25-1.35), BMLs (RR, 1.16-1.17), meniscal extrusion (RR, 1.22-1.33) and IPFP quality score (RR, 1.08-1.20) site-specifically and independently predicted an increase in MRI-detected OP ( $p$ values all $\leq 0.05$ ), after adjustment for covariates. Presence of IPFP abnormality was significantly associated with increased MRI-detected OPs but became non-significant after adjustment for other structural abnormalities. Total (RR, 1.27) and suprapatellar pouch effusion-synovitis (RR, 1.22) were both associated with increased MRI-detected OPs in the lateral compartment only (both $p<0.04)$.

Conclusion: Knee MRI-detected OPs are common in older adults and are likely to progress. The association between baseline structural abnormalities and worsening MRI-detected OPs suggest MRI-detected OP could be a consequence of multiple knee structural abnormalities.

Keywords: Osteoarthritis, Magnetic resonance imaging, Osteophyte, Natural history

\section{Background}

Osteoarthritis (OA) is a major contributor to overall disability in Western populations. Osteophytes (OPs) have long been viewed as a defining structural feature of knee OA [1] and a fundamental sign of disease incidence and progression [2]. They are associated with radiographic joint space narrowing, subchondral sclerosis, and cartilage defects in both tibiofemoral and

\footnotetext{
* Correspondence: Graeme.Jones@utas.edu.au

${ }^{2}$ Menzies Institute for Medical Research, University of Tasmania, Hobart, TAS Australia

Full list of author information is available at the end of the article
}

patellofemoral compartments [3, 4]. The size and extent of OP formation is routinely used for classifying the stage of OA [5].

Conventional radiographs remain the gold standard for assessment of OPs [6], but the association between presence of knee OPs on radiographs and symptoms is poor [7]. Two-dimensional conventional radiographs may miss the extent and size of OPs [8]. On the other hand, magnetic resonance imaging (MRI) is a non-invasive multiplanar tomographic modality that can assess many knee osteoarthritic changes [9]. Studies suggest that MRI can assess OPs

(c) The Author(s). 2018 Open Access This article is distributed under the terms of the Creative Commons Attribution 4.0 International License (http://creativecommons.org/licenses/by/4.0/), which permits unrestricted use, distribution, and 
with much greater sensitivity than radiographs and in locations that are not easily visualised by conventional radiography due to its ability to provide three-dimensional information [10-12]. Our recent study reported that MRI-detected OPs are highly prevalent in older adults (85\% vs $10 \%$ radiographic OPs prevalence) and can independently and site-specifically predict increases in cartilage defects, BMLs and loss of cartilage volume, and worsening knee pain over time, suggesting MRI-detected OPs are clinically relevant [13].

As far as we know, there are seldom longitudinal studies [14-16] investigating the relationship between MRI-detected OPs and clinical changes of OA. Sowers et al. found that large MRI-detected OPs were associated with increased odds of knee pain and reduced physical function [14]. Hakky et al. measured OP volume using MRI and observed significant positive correlation between OP volume and cartilage thickness loss [16]. In the latest study, MRI-detected OPs in a group of patients with end-stage OA scored using the Whole-Organ Magnetic Resonance Imaging Score (WORMS) grading system, those with a MRI-detected OP score of more than 30 have about threefold higher risk of undergoing total knee arthroplasty [15]. Based on the Chingford study, the natural history of radiographic $\mathrm{OA}$ is that of very slow progression [17]. However, the natural history of semi-quantitative MRI-detected OPs has not been described and it is unknown whether knee structural abnormalities, including cartilage defects, bone marrow lesions (BMLs), meniscal extrusion, infrapatellar fat pad (IPFP), and effusion-synovitis, can predict MRI-detected OP change over time. Hence, the aims of this study were to describe the natural history of knee MRI-detected OP, and to determine if knee structural abnormalities are associated with change of MRI-detected OP in a longitudinal study of older adults.

\section{Methods \\ Participants}

This study was from the Tasmania Older Adult Cohort (TASOAC) Study, a population-based, ongoing, prospective longitudinal cohort study which was designed to identify the genetic, environmental, and biochemical factors associated with the development and progression of $\mathrm{OA}$ at multiple sites. Participants between 50 and 80 years old were randomly selected from the electoral roll in Southern Tasmania (population 229,000) with an equal number of men and women (response rate 57\%). Participants were excluded if they were institutionalized or had contraindications to MRI. The Southern Tasmania Health and Medical Human Research Ethics Committee approved the study, and written informed consent was obtained from all participants. Baseline examinations were made between February 2002 and
September 2004, and follow-up measures were made at approximately 2.6 years later. This study consisted of 837 participants who had both knee MRI and radiographic scans at baseline.

\section{Anthropometrics}

Weight was measured using electronic scales (nearest $0.1 \mathrm{~kg}$ ), with shoes, socks and bulky clothing removed. Height was measured using a stadiometer (nearest $0.1 \mathrm{~cm}$ ), with shoes, socks and headgear removed. Body mass index (BMI) was calculated using height and weight $\left(\mathrm{kg} / \mathrm{m}^{2}\right)$.

\section{Magnetic resonance imaging}

MRI scans of the right knees were performed on two occasions and imaged in the sagittal plane on a 1.5-T whole body magnetic resonance unit (Picker, Cleveland, $\mathrm{OH}$, USA) using a commercial transmit-receive extremity coil. The image sequences used are listed as follows: (1) T1-weighted fat-saturation 3D gradient recall acquisition in the steady state; flip angle $30^{\circ}$; repetition time $31 \mathrm{~ms}$; echo time $6.71 \mathrm{~ms}$; field of view $16 \mathrm{~cm}$; 60 partitions; $512 \times 512$ matrix; acquisition time $11 \mathrm{~min} 56 \mathrm{~s}$; one acquisition. Sagittal images were obtained at a partition thickness of $1.5 \mathrm{~mm}$ and an in-plane resolution of $0.31 \times 0.31$ (512 $\times 512$ pixels); (2) T2-weighted fat-saturation 3D fast spin echo, flip angle 90, repetition time $3067 \mathrm{~ms}$, echo time $112 \mathrm{~ms}$, field of view $16 \mathrm{~cm}, 15$ partitions, $228 \times$ 256-pixel matrix; sagittal images were obtained at a partition thickness of $4 \mathrm{~mm}$ with a between-slices gap of 0.5 to $1.0 \mathrm{~mm}$. The image database was transferred to an independent computer workstation using the software program Osirix (University of Geneva, Geneva, Switzerland) as previously described $[18,19]$.

\section{MRI-detected osteophytes}

MRI-detected OPs were measured by ZZ using a combination of Whole-Organ Magnetic Resonance Imaging Score (WORMS) and the Knee Osteoarthritis Scoring System (KOSS) [9, 20]. OPs were defined as focal bony excrescences, seen on sagittal, axial or coronal images, extending from a cortical surface. Size was measured from the base (distinguished from that of adjacent articular cartilage with a normal MRI appearance) to the tip of the OP [11] at each of the following 14 sites: the anterior (a), central weight bearing (c) and posterior (p) margins of the femoral condyles and tibial plateaus, and the medial $(\mathrm{M})$ and lateral $(\mathrm{L})$ margins of the patella [20] (Fig. 1). OPs were graded as follows: grade 0 , absent; grade 1, minimal $(<3 \mathrm{~mm}$ high); grade 2 , moderate (3-5 $\mathrm{mm})$; grade 3 , severe $(>5 \mathrm{~mm})$ [9]. The sum total score of each individual site in the relevant compartment (or whole knee) was regarded as the OP score in that compartment (or whole knee). 

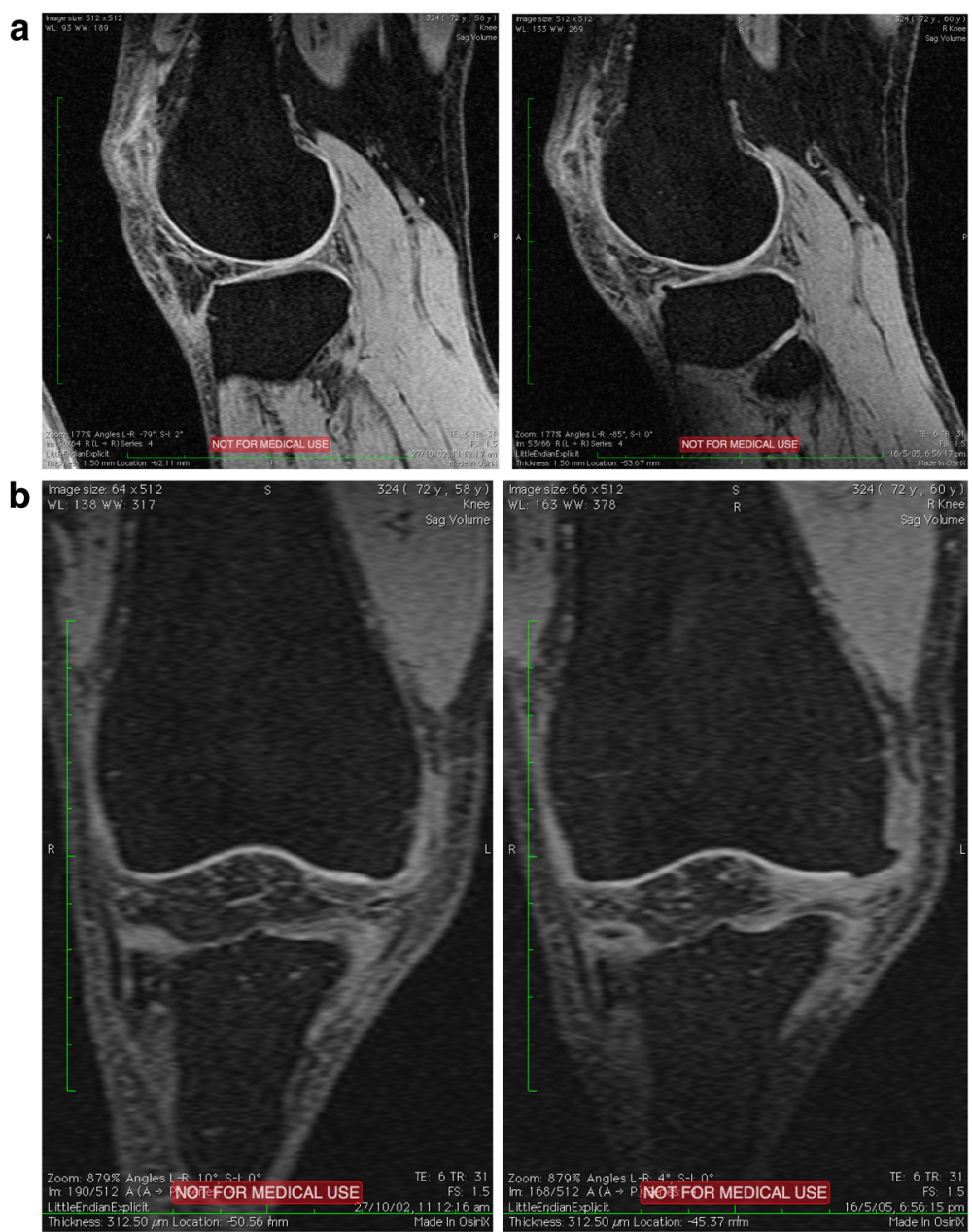

Fig. 1 Sample images show magnetic resonance imaging (MRI)-detected osteophyte (OP) progression. a Tibial MRI-detected OP increases from baseline to follow up (wider arrow indicates follow-up MRI-detected OP). b Femoral MRI-detected OP increases from baseline to follow up (wider arrow indicates follow-up MRI-detected OP)

MRI-detected OP score $\geq 1$ was considered as OP present. An increase in the MRI-detected OP score from baseline to follow up at any site was defined as a change of $\geq 1$ at the site. MRI-detected OPs were remeasured by $\mathrm{ZZ}$ and $\mathrm{WH}$ in 40 randomly selected participants, with a 4-week interval, to calculate intra-observer and inter-observer reliabilities. Intra-observer reliability (expressed as intraclass correlation coefficients, ICCs) was 0.94-0.97 and inter-observer reliability was $0.90-0.96[21]$.

\section{Cartilage defects}

Cartilage defects were graded by $\mathrm{CD}$ at the medial tibial, lateral tibial, medial femoral, lateral femoral and patellar regions as previously described [22] as follows: grade 0 , normal cartilage; grade 1 , focal blistering and low-signal intensity change with an intact surface and bottom; grade 2 , irregularities on the surface or bottom and loss of thickness
$<50 \%$; grade 4, full-thickness cartilage loss with exposure of subchondral bone [23]. The highest score of each individual site in the relevant compartment (or whole knee) was regarded as the cartilage defect score in that compartment (or whole knee). The presence of cartilage defects was defined as a cartilage defect score $\geq 2$ at any site. An increase in cartilage defects was defined as a change in cartilage defects $\geq 1$. Intra-observer reliability was $0.89-0.94$ and inter-observer reliability was $0.85-0.93[22]$.

\section{Cartilage volume}

Knee cartilage volume was measured on T1-weighted images by a single trained observer at baseline as previously described [23, 24]. The volumes of individual cartilage plates (medial tibial, lateral tibial, medial femoral, lateral femoral and patellar) were isolated from the total volume by manually drawing disarticulation contours around the cartilage boundaries on a section 
by section basis. These data were resampled by means of bilinear and cubic interpolation (area of $312 \times 312$ ) $\mu \mathrm{m}$ and $1.5 \mathrm{~mm}$ thickness, continuous sections) for the final $3 \mathrm{D}$ rendering. Changes in cartilage volume were calculated as:

Percentage change per annum $=[($ Follow-up volume Baseline volume)/Baseline cartilage volume]/Time between 2 scans in years $\times 100$.

The coefficients of variation $(\mathrm{CVs})$ for cartilage volume measures were $2.1 \%$ to $2.6 \%$ [23, 24].

\section{Bone marrow lesions}

Subchondral bone marrow lesions (BMLs) were defined as a discrete area of increased signal adjacent to the subcortical bone on T2-weighted MRI and were scored at the medial tibial, lateral tibial, medial femoral, lateral femoral, medial patellar and lateral patellar regions using a modified version of WORMS: grade 0 , absence of BML; grade 1 , area $<25 \%$ of the region; grade 2 , area between $25 \%$ and $50 \%$ of the region; grade 3 , area $>50 \%$ of the region [20]. The highest score of each individual site in the relevant compartment (or whole knee) was regarded as the BML score in that compartment (or whole knee). An increase in BMLs was defined as a change in BMLs $\geq 1$. The inter-observer reliability of this BML scoring system was assessed by randomly selecting 40 subjects with BMLs and having their MRI scans re-read by another observer. The ICCs for inter-observer reliability were also excellent $(0.73-0.95)[25,26]$.

\section{Meniscal extrusion}

Meniscal extrusion was assessed by a trained observer on T1-weighted MRI as previously described [27]. The proportion of the menisci affected by full extrusion was scored on the medial and lateral edges of the tibiofemoral joint space using a semi-quantitative scale. The extent of meniscal extrusion, not including the osteophytes, was evaluated for the anterior, middle and posterior horns of the menisci with 0 , no extrusion; 1, partial extrusion and 2, complete extrusion with no contact with the joint space (severe). The intra-observer and inter-observer correlation coefficient ranged from 0.85 to $0.92[28]$.

\section{Infrapatellar fat pad}

The infrapatellar fat pad (IPFP) was measured both semi-quantitatively and quantitatively. IPFP maximum area was measured by manually drawing disarticulation contours around the IPFP boundaries on a secton-by-section T2-weighted MR image, using the software program Osiris (University of Geneva). The maximal area was selected to represent the IPFP size. One observer graded the IPFP area in all MRI examinations. The intra-class correlation coefficient was 0.96 for intra-observer reliability and was 0.92 for inter-observer reliability [29]. The IPFP quality score was assessed semi-quantitatively according to its signal intensity alteration, which was defined as discrete areas of increased signal within IPFP: grade 0 , no signal intensity alteration; grade $1,<10 \%$ of the region has altered signal intensity; grade $2,10-20 \%$ of the region; grade $3,>20 \%$ of the region. Intra-observer and inter-observer reliability was high (intra-class correlation coefficient of 0.90 and inter-class correlation coefficient of 0.89 , respectively) [30]. Presence of IPFP abnormality was defined as a signal intensity alteration score $\geq 1$.

\section{Effusion-synovitis}

Knee effusion-synovitis at baseline was defined as the presence of intra-articular fluid-equivalent signal on T2-weighted MRI. We measured effusion-synovitis in the following subregions: (1) suprapatellar pouch, extending superiorly from the upper surface of the femur; (2) central portion, lying between the central femoral and tibia condyles, around the ligaments and menisci; (3) posterior femoral recess, lying behind the posterior portion of each femoral condyle and the deep surface of the lateral and medial heads of the gastrocnemius; (4) subpopliteal recess, lying posteriorly between the lateral meniscus and the popliteal tendon. Effusion-synovitis in each subregion was scored from 0 to 3 in terms of the estimated maximal distention of the synovial cavity: 0 , normal; $1<33 \%$ of maximum potential distention; $2,33-66 \%$ of maximum potential distention; $3,>66 \%$ of maximum potential distention [20]. The two independent observers scored images blinded to participant information. The intra-class reliability and the inter-class reliability in different subregions was $0.63-0.79$ [31].

\section{Statistical analysis}

Student's $t$ test or the chi-square $\left(\chi^{2}\right)$ test was used to compare means or proportions between participants with or without an increase in total knee MRI-detected OPs. The paired $t$ test was used to compare means between baseline and follow-up MRI-detected OPs in different compartments. Crude and adjusted log binominal regression was used to examine the longitudinal associations between increases in MRI-detected OPs (dependent variable), and baseline knee BMLs, cartilage defects, meniscal extrusion, effusion-synovitis and IPFP (independent variables), with age, sex, BMI and all the structural abnormalities as covariates. All statistical analyses were performed in Stata version 12.0 for Windows (StataCorp, College Station, TX, USA) [12]. A $p$ value < 0.05 (two-tailed) or a 95\% confidence interval (CI) not including a value of 1.00 was considered statistically significant. 


\section{Results}

\section{Characteristics of the study population}

A total of 1099 participants aged between 51 and 81 (mean 63) years were recruited to the TASOAC study of whom 837 had radiographs and MRI scans taken at baseline. The current study consists of a sample of 413 participants who had completed MRI scans at baseline and follow up. MRI scans were discontinued after this follow up due to decommissioning of the scanner. As reported previously [13], participants who did not complete follow-up MRI measures were similar to the remainder of the cohort in terms of demographics, smoking status, cartilage defects, BMLs, cartilage volume and radiographic OA at baseline. The characteristics of participants grouped by whether they had an increase or no increase in total knee MRI-detected OPs over 2.6 years of follow up are shown in Table 1. Participants with an increase in MRI-detected OPs over 2.6 years had significantly higher baseline BMI, MRI-detected OP score, cartilage defect score, BML score, meniscal extrusion score, total and suprapatellar effusion-synovitis score and IPFP quality score.

\section{Natural history of MRI-detected OPs}

The changes in MRI-detected OP scores by site over 2.6 years are presented in Fig. 2: 413 participants had completed follow-up MRI scans, 83\%, 69\%, 77\%, and $53 \%$ of these participants had stable MRI-detected OP scores in the medial tibiofemoral, lateral tibiofemoral, patellar and total knee, respectively. Of these participants, $17 \%, 30 \%, 23 \%$ and $46 \%$ had increased MRI-detected OP scores in the medial tibiofemoral, lateral tibiofemoral, patellar and total knee, respectively. Of the 413 participants (86\%) who completed follow up, 356 had knee MRI-detected OPs at baseline. Of these participants, $48 \%$ persisted in total MRI-detected OP size, $51 \%$ increased in total MRI-detected OP size, and only $1 \%$ decreased in total MRI-detected OP size. At baseline, MRI-detected OPs were absent in a total of 57 participants, of whom 11 (19\%) developed new OPs. Over 2.6 years, the average MRI-detected OP scores in all compartments increased significantly (Fig. 3).

\section{Factors associated with an increase in MRI-detected OPs}

Table 2 gives the association between baseline BMLs, cartilage defects, meniscal extrusion and increases in MRI-detected OPs over 2.6 years. Higher baseline BML scores and meniscal extrusion scores in medial tibiofemoral, lateral tibiofemoral and total compartments were significantly associated with increased MRI-detected OPs in the corresponding compartments, after adjustment for age, sex and BMI. These associations remained after further adjustment for baseline MRI-detected OPs and other structural abnormalities, except for the

Table 1 Characteristics of participants at baseline

\begin{tabular}{|c|c|c|c|}
\hline & Stable or decreased OPs & Increased OPs & $p$ \\
\hline & $N=222$ & $N=191$ & \\
\hline Age (years) & $62.2 \pm 7.3$ & $62.9 \pm 7.0$ & 0.30 \\
\hline Female (\%) & 53 & 47 & 0.22 \\
\hline BMI $\left(\mathrm{kg} / \mathrm{m}^{2}\right)$ & $26.9 \pm 3.9$ & $28.5 \pm 4.9$ & $<0.01$ \\
\hline Baseline total cartilage defect score (0-4) & $1.48 \pm 0.73$ & $2.13 \pm 1.03$ & $<0.01$ \\
\hline Baseline total BML score (0-3) & $0.33 \pm 0.56$ & $0.59 \pm 0.78$ & $<0.01$ \\
\hline Baseline total tibial CV (ml) & $5.11 \pm 1.22$ & $5.08 \pm 1.13$ & 0.80 \\
\hline Baseline meniscal extrusion score (0-2) & $0.13 \pm 0.36$ & $0.27 \pm 0.51$ & $<0.01$ \\
\hline Total effusion-synovitis score (0-3) & $1.90 \pm 0.67$ & $2.08 \pm 0.81$ & 0.01 \\
\hline Suprapatellar effusion-synovitis score (0-3) & $1.53 \pm 0.64$ & $1.76 \pm 0.84$ & 0.01 \\
\hline Central portion effusion-synovitis score (0-3) & $1.56 \pm 0.71$ & $1.65 \pm 0.77$ & 0.19 \\
\hline Posterior femoral recess effusion-synovitis score (0-3) & $0.66 \pm 0.79$ & $0.76 \pm 0.78$ & 0.14 \\
\hline Subpopliteal recess effusion-synovitis score (0-3) & $0.60 \pm 0.79$ & $0.78 \pm 0.94$ & 0.04 \\
\hline IPFP area $\left(\mathrm{cm}^{2}\right)$ & $7.6 \pm 1.2$ & $7.7 \pm 1.3$ & 0.18 \\
\hline Presence of IPFP abnormality (\%) & $75 \%$ & $82 \%$ & 0.10 \\
\hline IPFP quality score (0-6) & $1.45 \pm 1.21$ & $2.12 \pm 1.64$ & $<0.01$ \\
\hline Baseline total OP score (0-36) & $2.80 \pm 3.45$ & $7.62 \pm 7.70$ & $<0.01$ \\
\hline
\end{tabular}

The two-tailed $t$ test was used to examine differences between means, and the $\mathrm{x} 2$ test was used for proportions (percentages). Significant differences are shown in bold. Mean \pm SD except for percentages

$B M I$ body mass index, $B M$ : bone marrow lesion, $O P$ osteophyte, $C V$ cartilage volume, IPFP infrapatellar fat pad 


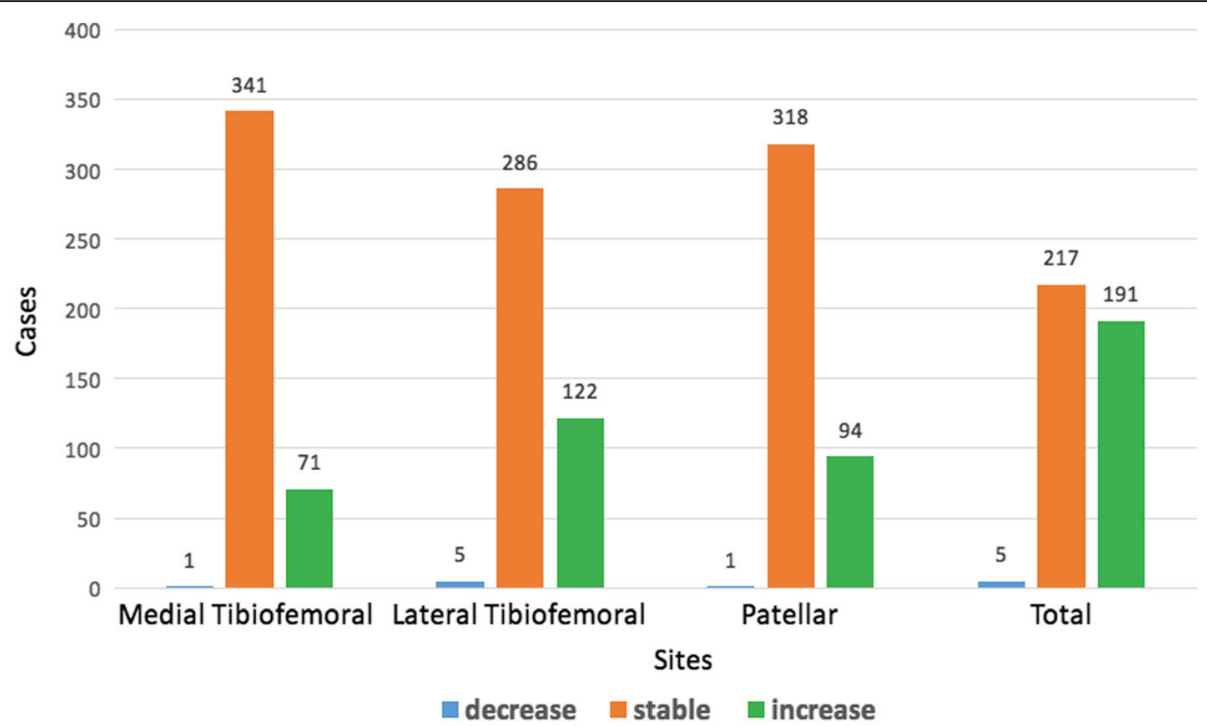

Fig. 2 Change in magnetic resonance imaging (MRI)-detected osteophyte (OP) scores by site over 2.6 years. Total score was calculated by summing medial tibiofemoral (tibfem), lateral tibiofemoral and patellar scores

associations with BMLs in the lateral tibiofemoral and patellar compartments. Higher baseline cartilage defect score in the medial tibiofemoral, lateral tibiofemoral and total compartments was significantly associated with increased MRI-detected OPs in the corresponding compartments, after adjustment for age, sex and BMI, and remained significant after further adjustment for baseline MRI-detected OPs and other structural abnormalities.

Table 3 shows the associations between baseline effusion-synovitis, IPFP and increased MRI-detected OPs over 2.6 years. Baseline total and suprapatellar effusion-synovitis scores were significantly associated with increases in total MRI-detected OP after adjustment for age, sex and BMI, but this did not persist after further adjustment for baseline OPs, BMLs and cartilage defects. Baseline effusion-synovitis score was not associated with increased medial MRI-detected OPs. In the lateral tibiofemoral compartment, baseline total, suprapatellar and central portion effusion-synovitis were significantly associated with increased MRI-detected OPs after adjustment for age, sex and BMI. The associations with total effusion-synovitis and suprapatellar effusion-synovitis but not central portion effusion-synovitis remained after further adjustment.

Baseline IPFP quality scores were significantly associated with increases in total MRI-detected OPs both before and after adjustment for covariates. In the medial tibiofemoral compartment, baseline IPFP quality score and presence of

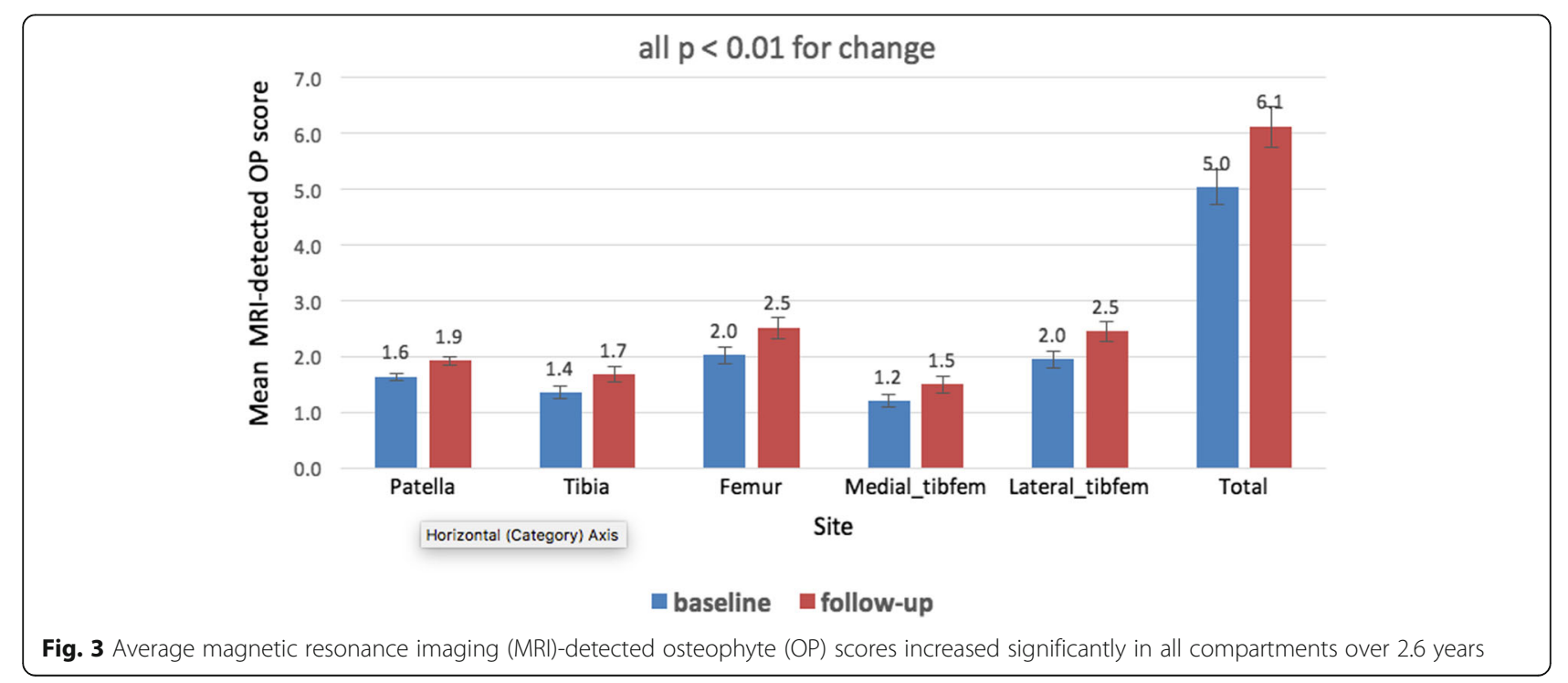


Table 2 Site-specific association with increase in MRI-detected OPs over 2.6 years

\begin{tabular}{|c|c|c|}
\hline & Multivariable* & Multivariable ${ }^{* *}$ \\
\hline & RR $(95 \% \mathrm{Cl})$ & RR $(95 \% \mathrm{Cl})$ \\
\hline \multicolumn{3}{|c|}{ Increase in MRI-detected OPs (yes or no) } \\
\hline Medial BMLs & $1.37(1.19,1.58)$ & $1.17(1.02,1.35)$ \\
\hline Lateral BMLs & $1.81(1.11,2.97)$ & $1.11(0.84,2.40)$ \\
\hline Patellar BMLs & $1.16(1.02,1.33)$ & $1.14(0.94,1.37)$ \\
\hline Total BMLs & $1.30(1.19,1.42)$ & $1.16(1.03,1.31)$ \\
\hline Medial cartilage defects & $1.39(1.25,1.54)$ & $1.30(1.16,1.45)$ \\
\hline Lateral cartilage defects & $1.42(1.26,1.61)$ & $1.35(1.21,1.51)$ \\
\hline Patellar cartilage defects & $1.34(1.22,1.47)$ & $1.25(1.13,1.38)$ \\
\hline Total cartilage defects & $2.19(1.71,2.80)$ & $1.33(1.20,1.46)$ \\
\hline Medial meniscal extrusion & $1.39(1.12,1.71)$ & $1.24(1.00,1.55)$ \\
\hline Lateral meniscal extrusion & $1.50(1.27,1.79)$ & $1.33(1.14,1.56)$ \\
\hline Total meniscal extrusion & $1.40(1.17,1.69)$ & $1.22(1.01,1.47)$ \\
\hline
\end{tabular}

Dependent variable: increases in magnetic resonance imaging (MRI)-detected osteophytes (OPs) (yes or no) in the same compartment as each exposure. Independent variables: baseline bone marrow lesions (BMLs), cartilage defects, meniscal extrusion (per grade)

*Adjusted for age, sex and body mass index

**Further adjusted for other structural abnormalities and baseline OP scores. Bold denotes statistical significance

IPFP abnormality was significantly associated with increased MRI-detected OPs after adjustment for age, sex and BMI, but only IPFP quality score remained significant after further adjustments. IPFP quality scores and presence of IPFP abnormality were significantly associated with increased MRI-detected OPs in the lateral tibiofemoral compartment after adjustment for age, sex and BMI, but only IPFP quality scores persisted after further adjustment. IPFP maximum area was not associated with increased MRI-detected OPs in any compartments (Table 3).

\section{Discussion}

To the best of our knowledge, this is the first study to describe the natural history of MRI-detected OPs and structural factors associated with this change. In this older adult sample, MRI-detected OPs were common, progressed in nearly half of participants over 2.6 years and rarely regressed. Baseline BMLs, cartilage defects, meniscal extrusion, presence of IPFP abnormality and effusion-synovitis were associated with worsening MRI-detected OPs, suggesting MRI-detected OPs are consequences of knee structural abnormalities.

Previous studies showed that knee MRI-detected OPs are far more common than OPs detected by conventional radiographs. One study reported that MRI-detected OPs were present in 60\% of older persons without radiographic OA [32], and another found that the prevalence of MRI-detected OPs was $72 \%$ among middle-aged women [14]. Our current study is largely in line with these studies, with MRI-detected OPs present in $85 \%$ of a community-based older population. We also found that $51 \%$ of those participants who had MRI-detected OPs at baseline progressed over time, compared with only $19 \%$ of those with no MRI-detected OPs at baseline. Hart et al. investigated the natural history of grade- 1 OPs measured by radiography in a 10 -year follow-up knee study and reported that $62 \%$ of participants graded at baseline with a "doubtful" OP went on to develop confirmed radiographic knee OA compared with only $22 \%$ of controls with no sign of disease [33]. In our current cohort, $46 \%$ of all participants had increased total knee MRI-detected OPs over 2.6 years. Leyland et al. reported the annual cumulative incidence of radiographic knee OA was $2.3 \%$ between baseline and year 15, and participants with a Kellgren-Lawrence score of 1 were four times more likely to experience worsening by year 15 compared with participants with a baseline grade 0 [17]. These findings indicate that early OP formation on radiographs can be used as an early marker of initiation of disease process and when identified by primary healthcare provider, should warrant further action. It is reasonable to hypothesize that the same is true for MRI-detected OP based on this paper and our recent report [13]. The apparently higher rate of change in MRI-detected OPs raises the possibility of MRI being used instead of x-rays for monitoring OP progression.

Correlation between cartilage damage and MRI-defined OPs has been reported previously [34]. But few other studies have examined the associations of IPFP abnormality and effusion-synovitis with MRI-detected OP progression. One cross-sectional study suggested, unsurprisingly, that greater size of MRI-detected OPs related to severity of radiographic OA [32]. Another cross-sectional study revealed that MRI-detected OPs were weakly associated with synovitis or joint effusion but not correlated with Kellgren-Lawrence score [35]. Hill et al. reported that change in synovitis correlated with change in knee pain, but not loss of cartilage [36]. The only longitudinal study to be published revealed significant associations between MRI-detected OP volume and cartilage thickness loss but did not investigate associations with other structures [16]. In the current study, baseline BMLs, cartilage defects, meniscal extrusion, IPFP abnormality and effusion-synovitis were associated with worsening MRI-detected OPs over time, but presence of IPFP abnormality, IPFP maximum area, effusion-synovitis in the central portion, posterior femoral recess and subpopliteal recess were not independently associated with worsening MRI-detected OPs over time. Although the underlying structural mechanisms are largely unknown, these findings reinforce the evolving concept that knee OA is a 
Table 3 Associations between baseline effusion-synovitis/IPFP and increased MRI-detected OPs over 2.6 years

\begin{tabular}{|c|c|c|}
\hline & Multivariable* & Multivariable** \\
\hline & $\mathrm{RR}(95 \% \mathrm{Cl})$ & RR $(95 \% \mathrm{Cl})$ \\
\hline \multicolumn{3}{|l|}{ Increased total MRI-detected OP (yes or no) } \\
\hline Total effusion-synovitis & $1.23(1.05,1.45)$ & $1.08(0.94,1.24)$ \\
\hline Suprapatellar effusion-synovitis & $1.25(1.09,1.45)$ & $1.08(0.92,1.64)$ \\
\hline Central portion effusion-synovitis & $1.11(0.96,1.29)$ & $1.06(0.94,1.20)$ \\
\hline Posterior femoral recess effusion-synovitis & $1.14(0.97,1.33)$ & $1.05(0.93,1.19)$ \\
\hline Subpopliteal recess effusion-synovitis & $1.15(1.03,1.29)$ & $1.07(0.97,1.18)$ \\
\hline IPFP quality score (0-6) & $1.17(1.10,1.24)$ & $1.08(1.01,1.15)$ \\
\hline IPFP maximum area $\left(\mathrm{cm}^{2}\right)$ & $1.04(0.93,1.16)$ & $1.03(0.93,1.14)$ \\
\hline IPFP abnormal change (yes/no) & $1.21(0.91,1.60)$ & $0.91(0.69,1.22)$ \\
\hline \multicolumn{3}{|l|}{ Increased medial MRI-detected OP (yes or no) } \\
\hline Total effusion-synovitis & $1.25(0.91,1.73)$ & $1.13(0.84,1.51)$ \\
\hline Suprapatellar effusion-synovitis & $1.31(0.96,1.80)$ & $1.15(0.89,1.48)$ \\
\hline Central portion effusion-synovitis & $1.14(0.83,1.58)$ & $1.02(0.75,1.39)$ \\
\hline Posterior femoral recess effusion-synovitis & $1.10(0.83,1.46)$ & $1.01(0.76,1.33)$ \\
\hline Subpopliteal recess effusion-synovitis & $1.22(0.99,1.52)$ & $1.09(0.88,1.34)$ \\
\hline IPFP quality score (0-6) & $1.40(1.24,1.57)$ & $1.20(1.06,1.37)$ \\
\hline IPFP maximum area $\left(\mathrm{cm}^{2}\right)$ & $0.96(0.77,1.20)$ & $0.96(0.78,1.19)$ \\
\hline IPFP abnormal change (yes/no) & $3.17(1.31,7.65)$ & $1.87(0.73,4.79)$ \\
\hline \multicolumn{3}{|l|}{ Increased lateral MRI-detected OP (yes or no) } \\
\hline Total effusion-synovitis & $1.40(1.13,1.75)$ & $1.27(1.03,1.57)$ \\
\hline Suprapatellar effusion-synovitis & $1.37(1.22,1.68)$ & $1.22(1.01,1.47)$ \\
\hline Central portion effusion-synovitis & $1.24(1.00,1.53)$ & $1.16(0.97,1.40)$ \\
\hline Posterior femoral recess effusion-synovitis & $1.18(0.97,1.43)$ & $1.08(0.91,1.28)$ \\
\hline Subpopliteal recess effusion-synovitis & $1.12(0.96,1.31)$ & $1.08(0.94,1.25)$ \\
\hline IPFP quality score (0-6) & $1.29(1.19,1.40)$ & $1.19(1.08,1.30)$ \\
\hline IPFP maximum area $\left(\mathrm{cm}^{2}\right)$ & $1.02(0.89,1.18)$ & $1.05(0.91,1.21)$ \\
\hline IPFP abnormal change (yes/no) & $1.87(1.15,3.03)$ & $1.40(0.87,2.25)$ \\
\hline
\end{tabular}

whole-organ disease and that most structures are involved, including cartilage, meniscal, effusion-synovitis, subchondral bone and IPFP.

This study also found that participants with higher BMI had a higher rate of OP progression, which is in line with previous studies $[37,38]$. The increased loading may have altered the biomechanics of the knee joints and accelerated the progression of OP, while a sedentary lifestyle may contribute to rapid progression of both BMI and OPs [39].

Although cartilage damage and OP formation are not perfectly correlated [40], joint space narrowing, which is a surrogate of cartilage damage, has been reported to be highly associated with the presence of OPs [41]. In animal models, OPs have been found to develop at sites of adjacent cartilage loss [42]. This is consistent with the current finding that cartilage defects at baseline site-specifically predicted an increase in MRI-detected OPs over 2.6 years.

In an older population sample, a fair proportion (61\%) of the participants with meniscal abnormalities had no knee pain [43]. However, meniscal pathology is associated with the development of radiographic OA [44-46]. The existence of meniscal damage in a compartment appears to be a factor affecting the progression of OPs. Moreover, a study suggested that the mechanical stimuli in certain compartments may translate to factors on a cellular level or autonomous biochemical stimuli that initiate the process of OP formation [40, 47]. Felson et al. suggested that OPs are not directly involved in 
disease progression but might serve as markers of the location and severity of the pathologic process [8]. Our study found that BMLs, cartilage defects, meniscal extrusion, IPFP abnormality and effusion-synovitis were the structural risk factors for worsening MRI-detected OP, suggesting that MRI-detected OP could be a result of other osteoarthritic structural abnormalities. On the other hand, it should be noted that MRI-detected OPs were also found to consistently and independently predict changes in knee cartilage, BMLs and cartilage volume, and the need to undergo total knee replacement (TKA) $[13,15]$. It is thought that subchondral bone expansion leads to splitting of cartilage and is potentially a precursor to formation of cartilage defects [22, 48]. Combined with our current findings, these studies indicate that OP formation is involved in the OA disease pathway and can be both a risk factor and a consequence of knee OA progression, suggesting the processes are not independent but are linked.

A combination of WORMS and KOSS for the measurement of OPs was employed in the present study. These two grading systems are validated instruments, which have good reliability to assess OPs semi-quantitatively on MRI $[9,20]$. The WORMS grading system has the advantage of subdividing the whole knee into different subregions, which includes both marginal and central OPs, but its OP grading scale is more subjective. On the other hand, the KOSS grading system has the advantage of a quatitative OP grading scale for each subregion. The reliability of our measures was excellent.

Strengths of this study included the random selection of participants for the cohort from a community, with a large sample size. On the other hand, there are some potential limitations in our study. First, follow-up MRI scans were only available in 413 out of 837 participants due to decommissioning of the MRI scanner. However, there were no significant differences in demographic factors, ROA, baseline cartilage defects and BMLs between the current study sample and the rest of the cohort. Second, using a higher field-strength magnet than $1.5 \mathrm{~T}$ might be marginally more sensitive in detecting OPs; however, as reported previously [49], the results are unlikely to be markedly different as this benefit is modest. Third, the reproducibility for measurement of MRI-detected OPs was good rather than excellent, which may contribute to underestimation of the associations.

\section{Conclusion}

Knee MRI-detected OPs are common in older adults and are likely to progress. The associations between baseline structural abnormalities and worsening MRI-detected OPs suggest MRI-detected OPs could be a consequence of multiple knee structural abnormalities.

\section{Abbreviations}

BMI: Body mass index; BML: Bone marrow lesion; ICCs: Intraclass correlation coefficients; IPFP: Infrapatellar fat pad; KOSS: Knee Osteoarthritis Scoring System; MRI: Magnetic resonance imaging; OA: Osteoarthritis;

OP: Osteophyte; ROA: Radiographic osteoarthritis; TASOAC: Tasmania Older Adult Cohort Study; TKA: Total knee arthroplasty; WORMS: Whole-Organ Magnetic Resonance Imaging Score

\section{Acknowledgements}

The authors thank the participants who made this study possible, and acknowledge the role of the staff and volunteers in collecting the data, particularly research nurses Boon C and Boon P. Warren R assessed MRIs and Dr Srikanth $\mathrm{V}$ and Dr Cooley $\mathrm{H}$ assessed radiographs.

\section{Patient consent}

Obtained.

\section{Funding}

This study was funded by the National Health and Medical Research Council of Australia (302204), the Tasmanian Community Fund (D0015018), the Arthritis Foundation of Australia (MRI06161) and the University of Tasmania Institutional Research Grants Scheme (D0015019).

\section{Availability of data and materials}

The datasets generated and/or analyzed during the current study are available from the corresponding author on reasonable request. Data generated or analyzed during this study are included in this published article or are noted as data not shown.

\section{Authors' contributions}

ZZ had full access to all the data in the study and takes responsibility for the integrity of the data and the accuracy of the data analysis. Study design: FC and GJ. Acquisition of data: ZZ, CD, LL, WH and GJ. Analysis and interpretation of data: ZZ, LL, WH, SZ, FC, TW, GJ and CD. Manuscript preparation and approval: ZZ, LL, WH, SZ, FC, TW, GJ and CD.

\section{Ethics approval and consent to participate}

This study was approved by the Southern Tasmania Health and Medical Human Research Ethics Committee, and written informed consent was obtained from all participants.

\section{Consent for publication}

Not applicable.

\section{Competing interests}

The authors declare that they have no competing interests.

\section{Publisher's Note}

Springer Nature remains neutral with regard to jurisdictional claims in published maps and institutional affiliations.

\section{Author details}

${ }^{1}$ Clinical Research Centre, Zhujiang Hospital, Southern Medical University, Guangzhou, Guangdong, China. ${ }^{2}$ Menzies Institute for Medical Research, University of Tasmania, Hobart, TAS, Australia. ${ }^{3}$ Department of Epidemiology and Preventive Medicine, Monash University, Melbourne, VIC, Australia.

${ }^{4}$ Faculty of Health, University of Tasmania, Hobart, TAS, Australia.

Received: 12 August 2018 Accepted: 25 September 2018

Published online: 23 October 2018

\section{References}

1. Spector TD, Hart DJ, Byrne J, Harris PA, Dacre JE, Doyle DV. Definition of osteoarthritis of the knee for epidemiological studies. Ann Rheum Dis. 1993; 52(11):790-4.

2. Felson DT, McAlindon TE, Anderson JJ, Naimark A, Weissman BW, Aliabadi P, Evans S, Levy D, LaValley MP. Defining radiographic osteoarthritis for the whole knee. Osteoarthr Cartil. 1997;5(4):241-50.

3. Boegard T, Rudling O, Petersson IF, Jonsson K. Correlation between radiographically diagnosed osteophytes and magnetic resonance detected cartilage defects in the tibiofemoral joint. Ann Rheum Dis. 1998;57(7):401-7. 
4. Dieppe PA, Cushnaghan J, Shepstone L. The Bristol 'OA500' study: progression of osteoarthritis $(\mathrm{OA})$ over 3 years and the relationship between clinical and radiographic changes at the knee joint. Osteoarthr Cartil. 1997; 5(2):87-97.

5. Kellgren JH, Lawrence JS. Radiological assessment of osteo-arthrosis. Ann Rheum Dis. 1957;16(4):494-502.

6. Lanyon P, O'Reilly S, Jones A, Doherty M. Radiographic assessment of symptomatic knee osteoarthritis in the community: definitions and normal joint space. Ann Rheum Dis. 1998;57(10):595-601.

7. Eckstein F, Wirth W, Hudelmaier MI, Maschek S, Hitzl W, Wyman BT, Nevitt M, Le Graverand MPH, Hunter D, OA Initiative Investigator Group. Relationship of compartment-specific structural knee status at baseline with change in cartilage morphology: a prospective observational study using data from the osteoarthritis initiative. Arthritis Res Ther. 2009;11(3):R90

8. Felson DT, Gale DR, Elon Gale M, Niu J, Hunter DJ, Goggins J, Lavalley MP. Osteophytes and progression of knee osteoarthritis. Rheumatology (Oxford). 2005:44(1):100-4.

9. Kornaat PR, Ceulemans RYT, Kroon HM, Riyazi N, Kloppenburg M, Carter WO, Woodworth TG, Bloem JL. MRI assessment of knee osteoarthritis: Knee Osteoarthritis Scoring System (KOSS) - inter-observer and intra-observer reproducibility of a compartment-based scoring system. Skelet Radiol. 2005;34(2):95-102.

10. Kwok WY, Kortekaas MC, Reijnierse M, van der Heijde D, Bloem JL, Kloppenburg M. MRI in hand osteoarthritis: validation of the Oslo Hand Osteoarthritis MRI-Scoring Method and association with pain. Osteoarthr Cartilage. 2011;19:526-7.

11. McCauley TR, Kornaat PR, Jee WH. Central osteophytes in the knee: prevalence and association with cartilage defects on MR imaging. Am J Roentgenol. 2001;176(2):359-64.

12. Katsuragi J, Sasho T, Yamaguchi S, Sato Y, Watanabe A, Akagi R, Muramatsu Y, Mukoyama S, Akatsu Y, Fukawa T, et al. Hidden osteophyte formation on plain $x$-ray is the predictive factor for development of knee osteoarthritis after 48 months - data from the Osteoarthritis Initiative. Osteoarthr Cartilage. 2015;23(3):383-90.

13. Zhu Z, Laslett LL, Jin X, Han W, Antony B, Wang X, Lu M, Cicuttini F, Jones G, Ding C. Association between MRI-detected osteophytes and changes in knee structures and pain in older adults: a cohort study. Osteoarthr Cartilage. 2017; 25(7):1084-92.

14. Sowers M, Karvonen-Gutierrez CA, Jacobson JA, Jiang Y, Yosef M. Associations of anatomical measures from MRI with radiographically defined knee osteoarthritis score, pain, and physical functioning. J Bone Joint Surg Am. 2011;93(3):241-51

15. Liu L, Ishijima M, Kaneko H, Sadatsuki R, Hada S, Kinoshita M, Aoki T, Futami I, Yusup A, Arita H, et al. The MRl-detected osteophyte score is a predictor for undergoing joint replacement in patients with end-stage knee osteoarthritis. Mod Rheumatol. 2017;27(2):332-8.

16. Hakky M, Jarraya M, Ratzlaff C, Guermazi A, Duryea J. Validity and responsiveness of a new measure of knee osteophytes for osteoarthritis studies: data from the Osteoarthritis Initiative. Osteoarthr Cartil. 2015;23(12): 2199-205.

17. Leyland KM, Hart DJ, Javaid MK, Judge A, Kiran A, Soni A, Goulston LM, Cooper C, Spector TD, Arden NK. The natural history of radiographic knee osteoarthritis: a fourteen-year population-based cohort study. Arthritis Rheum. 2012;64(7):2243-51.

18. Peterfy CG, Vandijke CF, Janzen DL, Gluer CC, Namba R, Majumdar S, Lang P, Genant HK. Quantification of articular-cartilage in the knee with pulsed saturation-transfer subtraction and fat-suppressed Mr-imaging - optimization and validation. Radiology. 1994;192(2):485-91.

19. Jones $G$, Ding $C H$, Scott F, Glisson M, Cicuttini F. Early radiographic osteoarthritis is associated with substantial changes in cartilage volume and tibial bone surface area in both males and females. Osteoarthr Cartilage. 2004;12(2):169-74.

20. Peterfy CG, Guermazi A, Zaim S, Tirman PFJ, Miaux Y, White D, Kothari M, Lu Y, Fye $K$, Zhao $S$, et al. Whole-organ magnetic resonance imaging score (WORMS) of the knee in osteoarthritis. Osteoarthr Cartilage. 2004;12(3):177-90.

21. Altman DG. Statistics in medical journals - developments in the 1980s. Stat Med. 1991;10(12):1897-913.

22. Ding $\mathrm{CH}$, Garnero P, Cicuttini F, Scott F, Cooley H, Jones G. Knee cartilage defects: association with early radiographic osteoarthritis, decreased cartilage volume, increased joint surface area and type II collagen breakdown. Osteoarthr Cartilage. 2005;13(3):198-205.
23. Ding C, Cicuttini F, Scott F, Glisson M, Jones G. Sex differences in knee cartilage volume in adults: role of body and bone size, age and physical activity. Rheumatology. 2003;42(11):1317-23.

24. Jones G, Glisson M, Hynes K, Cicuttini F. Sex and site differences in cartilage development - a possible explanation for variations in knee osteoarthritis in later life. Arthritis Rheum-Us. 2000;43(11):2543-9.

25. Raynauld JP, Martel-Pelletier J, Berthiaume MJ, Abram F, Choquette D, Haraoui B, Beary JF, Cline GA, Meyer JM, Pelletier JP. Correlation between bone lesion changes and cartilage volume loss in patients with osteoarthritis of the knee as assessed by quantitative magnetic resonance imaging over a 24-month period. Ann Rheum Dis. 2008;67(5):683-8.

26. Wang J, Antony B, Zhu Z, Han W, Pan F, Wang X, Jin X, Liu Z, Cicuttini F, Jones $\mathrm{G}$, et al. Association of patellar bone marrow lesions with knee pain, patellar cartilage defect and patellar cartilage volume loss in older adults: a cohort study. Osteoarthr Cartilage. 2015;23(8):1330-6.

27. Berthiaume MJ, Raynauld JP, Martel-Pelletier J, Labonte F, Beaudoin G, Bloch DA, Choquette D, Haraoui B, Altman RD, Hochberg M, et al. Meniscal tear and extrusion are strongly associated with progression of symptomatic knee osteoarthritis as assessed by quantitative magnetic resonance imaging. Ann Rheum Dis. 2005;64(4):556-63.

28. Raynauld JP, Martel-Pelletier J, Berthiaume MJ, Beaudoin G, Choquette D, Haraoui B, Tannenbaum H, Meyer JM, Beary JF, Cline GA, et al. Long term evaluation of disease progression through the quantitative magnetic resonance imaging of symptomatic knee osteoarthritis patients: correlation with clinical symptoms and radiographic changes. Osteoporosis Int. 2006;17:S13.

29. Pan F, Han W, Wang X, Liu Z, Jin X, Antony B, Cicuttini F, Jones G, Ding C. A longitudinal study of the association between infrapatellar fat pad maximal area and changes in knee symptoms and structure in older adults. Ann Rheum Dis. 2015;74(10):1818-24.

30. Han W, Aitken D, Zhu Z, Halliday A, Wang X, Antony B, Cicuttini F, Jones G, Ding $C$. Hypointense signals in the infrapatellar fat pad assessed by magnetic resonance imaging are associated with knee symptoms and structure in older adults: a cohort study. Arthritis Res Ther. 2016;18(1):234.

31. Wang X, Blizzard L, Halliday A, Han W, Jin X, Cicuttini F, Jones G, Ding C. Association between MRI-detected knee joint regional effusion-synovitis and structural changes in older adults: a cohort study. Ann Rheum Dis. 2016;75(3):519-25.

32. Hayes CW, Jamadar DA, Welch GW, Jannausch ML, Lachance LL, Capul DC, Sowers MR. Osteoarthritis of the knee: comparison of MR imaging findings with radiographic severity measurements and pain in middle-aged women. Radiology. 2005;237(3):998-1007.

33. Hart DJ, Spector TD. Kellgren \& Lawrence grade 1 osteophytes in the knee-doubtful or definite? Osteoarthr Cartil. 2003;11(2):149-50.

34. Roemer FW, Guermazi A, Niu JB, Zhang YQ, Mohr A, Felson DT. Prevalence of magnetic resonance imaging-defined atrophic and hypertrophic phenotypes of knee osteoarthritis in a population-based cohort. Arthritis Rheum-Us. 2012;64(2):429-37.

35. Link TM, Steinbach LS, Ghosh S, Ries M, Lu Y, Lane N, Majumdar S. Osteoarthritis: MR imaging findings in different stages of disease and correlation with clinical findings. Radiology. 2003;226(2):373-81.

36. Hill CL, Hunter DJ, Niu J, Clancy M, Guermazi A, Genant H, Gale D, Grainger A, Conaghan P, Felson DT. Synovitis detected on magnetic resonance imaging and its relation to pain and cartilage loss in knee osteoarthritis. Ann Rheum Dis. 2007;66(12):1599-603.

37. Wills AK, Black S, Cooper R, Coppack RJ, Hardy R, Martin KR, Cooper C, Kuh D. Life course body mass index and risk of knee osteoarthritis at the age of 53 years: evidence from the 1946 British birth cohort study. Ann Rheum Dis. 2012;71(5):655-60.

38. Zheng $\mathrm{H}$, Chen $\mathrm{C}$. Body mass index and risk of knee osteoarthritis: systematic review and meta-analysis of prospective studies. BMJ Open. 2015;5(12):e007568.

39. Musumeci G, Aiello FC, Szychlinska MA, Di Rosa M, Castrogiovanni P, Mobasheri A. Osteoarthritis in the XXIst century: risk factors and behaviours that influence disease onset and progression. Int J Mol Sci. 2015;16(3):6093-112.

40. van der Kraan PM, van den Berg WB. Osteophytes: relevance and biology. Osteoarthr Cartil. 2007;15(3):237-44.

41. Boegard T, Rudling O, Petersson IF, Jonsson K. Correlation between radiographically diagnosed osteophytes and magnetic resonance detected cartilage defects in the patellofemoral joint. Ann Rheum Dis. 1998;57(7):395-400. 
42. vanOsch GJVM, vanderKraan PM, vanValburg AA, vandenBerg WB. The relation between cartilage damage and osteophyte size in a murine model for osteoarthritis in the knee. Rheumatol Int. 1996;16(3):115-9.

43. Englund M, Guermazi A, Gale D, Hunter DJ, Aliabadi P, Clancy M, Felson DT. Incidental meniscal findings on knee MRI in middle-aged and elderly persons. N Engl J Med. 2008;359(11):1108-15.

44. Sharma L, Chmiel JS, Almagor O, Dunlop D, Guermazi A, Bathon JM, Eaton CB, Hochberg MC, Jackson RD, Kwoh CK, et al. Significance of preradiographic magnetic resonance imaging lesions in persons at increased risk of knee osteoarthritis. Arthritis Rheumatol. 2014;66(7):1811-9.

45. Englund M, Niu J, Guermazi A, Roemer FW, Hunter DJ, Lynch JA, Lewis CE, Torner J, Nevitt MC, Zhang YQ, et al. Effect of meniscal damage on the development of frequent knee pain, aching, or stiffness. Arthritis Rheum. 2007;56(12):4048-54

46. van Oudenaarde K, Jobke B, Oostveen AC, Marijnissen AC, Wolterbeek R, Wesseling J, Bierma-Zeinstra SM, Bloem HL, Reijnierse M, Kloppenburg M. Predictive value of MRI features for development of radiographic osteoarthritis in a cohort of participants with pre-radiographic knee osteoarthritis-the CHECK study. Rheumatology (Oxford). 2017;56(1):113-20.

47. Blom AB, van Lent PL, Holthuysen AE, van der Kraan PM, Roth J, van Rooijen $N$, van den Berg WB. Synovial lining macrophages mediate osteophyte formation during experimental osteoarthritis. Osteoarthr Cartil. 2004;12(8):627-35.

48. Davies-Tuck ML, Wluka AE, Wang Y, Teichtahl AJ, Jones G, Ding C, Cicuttini FM. The natural history of cartilage defects in people with knee osteoarthritis. Osteoarthr Cartil. 2008;16(3):337-42.

49. Roemer FW, Lynch JA, Niu J, Zhang Y, Crema MD, Tolstykh I, El-Khoury GY, Felson DT, Lewis CE, Nevitt MC, et al. A comparison of dedicated 1.0 T extremity MRI vs large-bore $1.5 \mathrm{~T}$ MRI for semiquantitative whole organ assessment of osteoarthritis: the MOST study. Osteoarthr Cartil. 2010;18(2):168-74.

Ready to submit your research? Choose BMC and benefit from:

- fast, convenient online submission

- thorough peer review by experienced researchers in your field

- rapid publication on acceptance

- support for research data, including large and complex data types

- gold Open Access which fosters wider collaboration and increased citations

- maximum visibility for your research: over $100 \mathrm{M}$ website views per year

At $\mathrm{BMC}$, research is always in progress.

Learn more biomedcentral.com/submissions 